\title{
Characterization of clinical enterococci isolates, focusing on the vancomycin- resistant enterococci in a tertiary hospital in China: based on the data from 2013 to 2018
}

Wanqing Zhou ${ }^{1 \dagger}$, Hui Zhou ${ }^{1+}$, Yuhan Sun ${ }^{1}$, Shuo Gao ${ }^{1}$, Yan Zhang ${ }^{1}$, Xiaoli Cao ${ }^{1}$, Zhifeng Zhang ${ }^{1}$, Han Shen ${ }^{1 *}$ and Chunni Zhang ${ }^{2^{*}}$

\begin{abstract}
Background: Vancomycin-resistant Enterococcus spp. (VRE) have spread all over the world. The present study aims to investigate the species distribution, specimen type and susceptibilities of Enterococcal species collected from Nanjing Drum Tower Hospital from 2013 to 2018. Additionally, distribution of VRE and prevalence of van gene among VRE isolates were also analyzed.

Methods: The susceptibilities of 3913 Enterococcus isolates were retrospectively investigated. Among these strains, 60 VRE strains were further anazlyed in this study. The minimum inhibitory concentrations (MICs) of the VRE strains towards vancomycin, teicoplanin and linezolid were determined by E-test. Polymerase chain reaction (PCR) and DNA sequencing were used to investigate the prevalence of van genes among VRE. Furthermore, the sequence types (STs) of VRE strains were explored by multi-locus sequence typing (MLST).

Results: Among the 3913 enterococci isolates, Enterococcus faecalis ( $n=1870,47.8 \%)$ and Enterococcus faecium $(1738,44.4 \%)$ were the main isolates. These Enterococcus strains were mainly isolated from urine $(n=1673,42.8 \%)$, followed by secretions ( $n=583,14.9 \%)$ and ascites $(n=554,14.2 \%)$. VRE displayed a decreasing trend year by year. Molecular analysis revealed that 49 out of 60 VRE isolates carried vanA gene, 10 carried vanM, and 1 carried both vanA and vanM genes. Sixteen distinct STs were identified among the 58 VREM, with ST78 $(n=16)$, ST192 $(n=8)$ and ST570 ( $n=7)$ being the most dominant ones.

(Continued on next page)
\end{abstract}

\footnotetext{
* Correspondence: shenhan10366@sina.com; zchunni27@hotmail.com

†Wanqing Zhou and Hui Zhou contributed equally to this work.

${ }^{1}$ Department of Laboratory Medicine, Nanjing Drum Tower Hospital, The Affiliated Hospital of Nanjing University Medical School, 321\# Zhongshan Road, Gulou District, Nanjing, Jiangsu Province 210008, P. R. China

${ }^{2}$ Department of Clinical Laboratory, Jinling Hospital, Nanjing University School of Medicine, Nanjing University, 305\# East Zhongshan Road, Qinhuai District, Nanjing, Jiangsu Province 210008, P. R. China
}

(C) The Author(s). 2020 Open Access This article is licensed under a Creative Commons Attribution 4.0 International License, which permits use, sharing, adaptation, distribution and reproduction in any medium or format, as long as you give appropriate credit to the original author(s) and the source, provide a link to the Creative Commons licence, and indicate if changes were made. The images or other third party material in this article are included in the article's Creative Commons licence, unless indicated otherwise in a credit line to the material. If material is not included in the article's Creative Commons licence and your intended use is not permitted by statutory regulation or exceeds the permitted use, you will need to obtain permission directly from the copyright holder. To view a copy of this licence, visit http://creativecommons.org/licenses/by/4.0/ The Creative Commons Public Domain Dedication waiver (http://creativecommons.org/publicdomain/zero/1.0/) applies to the data made available in this article, unless otherwise stated in a credit line to the data. 
(Continued from previous page)

Conclusions: $E$. faecalis and E. faecium were the major enterococci strains which are the main pathogens of urinary traction infections; vanA and vanM were the main determinants conferring resistance to vancomycin; ST78, ST192 and ST570 were the leading STS of VREM which displayed a decreasing trend of prevalence year by year.

Keywords: Enterococcus, VRE, Enterococcus faecium, Enterococcus faecalis, vanA, vanM

\section{Background}

Enterococci are leading pathogens of nosocomial infections such as bacteraemia, intra-abdominal infections and surgical wounds, especially urinary tract infections [1]. With the antimicrobial agents being frequently used in clinical treatment, antibiotic-resistant enterococci, particularly multi-drug resistant enterococci isolates, such as vancomycin-resistant enterococci (VRE) and linezolidresistant enterococci (LRE) have emergence and spread all over the world $[2,3]$.

In Europe, an increasing proportion of vancomycinresistant E. faecium (VREM) has been reported [4], with the VREM being increased from $11.2 \%$ in 2014 to $26.1 \%$ in 2017 in German hospitals [5]; In Canadian hospitals, the national prevalence of VRE tripled from $1.8 \%$ in 2007 to $6.0 \%$ in 2013 and peaked at $7.9 \%$ in 2011 [6]; Thus, besides high cost of treatment of VRE, high mortality and additional morbidity caused by the frequent incidence of infections has been reported in US and European countries [1]. Whereas in China, Antimicrobial Surveillance Network (CHINET) showed a declining trend of VRE year by year during 2013 to 2018 all over the China (http://www.chinets.com/Data/GermYear). However, infections of VRE have lead to increased cost and mortality compared to vancomycin-susceptible Enterococcus isolates [7]. The similar situation has also been observed on the distribution of VRE in Zhejiang, China during the period of 2015-2017 [8]. However, data on the characterization of VRE in tertiary hospitals in China are still limited.

To date, treatment options for infections caused by VRE are quite limited, including vancomycin, teicoplanin, linezolid and fosfomycin [1]. However, ranges on the MIC determinations of VRE on these antimicrobial agents are still unclear, which is very important for choice of drugs in clinical therapy.

Moreover, it has been well known that resistance to vancomycin in Enterococci is mediated by van genes. To date, vanA, vanB, vanC, vanD, vanE, vanG, vanL, vanM, and $v a n N$ have been identified [9]. vanA and $v a n B$ genotypes have predominated worldwide [10] and are mainly identified in VREM and vancomycin-resistant E. faecalis (VREF) isolates. Whereas, vanM gene was initially identified in a VREM clinical isolate in Shanghai in 2006 [11], subsequently reported in Singapore [12]. Presently, vanM has been the predominant gene in VRE in
Shanghai since 2011 [13]. Noteworthiliy, albeit vanM gene has also been isolated in Hangzhou [14, 15], which is located approximately $200 \mathrm{~km}$ from Shanghai, and later in Beijing [16], it has not been detected in other cities in China. Thus, more data on the prevalence of VRE and the distribution of van genes among VRE in other cities of China are needed.

In this study, the strain types, sample sources, and susceptibilities of clinical enterococci collected in our hospital during 2013 to 2018 were retrospectively analyzed; Furthermore, the distribution of VRE strains, prevalence of van genes and sequence types (STs) among VRE were further investigated to analyze the characterization.

\section{Methods \\ Bacterial strains}

Consecutive and non-duplicate Enterococcus isolates were recovered in Nanjing Drum Tower Hospital (Nanjing, China) from January 2013 until December 2018 by WHONET 5.6 software. Confirmation of enterococci was performed using Vitek 2 Compact GP cards (bioMerieux, France). VRE were screened according to susceptibility to vancomycin (MIC $>=32 \mu \mathrm{g} / \mathrm{ml}$ ). Where Antimicrobial testing was performed using Vitek 2 Compact GP67 cards (bioMerieux, France) or the disc diffusion method on Mueller-Hinton agar (bioMerieux, France), according to Clinical and Laboratory Standards Institute guidelines (CLSI, 2018) [17].

\section{Antimicrobial susceptibility testing}

The minimum inhibitory concentrations (MICs) of VRE toward vancomycin, teicoplanin and linezolid were further determined by E-test (bioMerieux, France). The susceptibility of the VRE isolates to fosfomycin was tested by the disc diffusion method on Mueller-Hinton agar (bioMerieux, France). E. faecalis ATCC 29212 and $S$. aureus 29213 were used as quality controls in parallel. The antimicrobial susceptibility testing results were interpreted according to CLSI 2018 [17].

\section{DNA extraction and quantification}

DNA extraction was performed by boiling method. Briefly, fresh colonies were picked into $1 \mathrm{ml}$ sterile saline. After boiling for $10 \mathrm{~min}$ at $100{ }^{\circ} \mathrm{C}$, followed by centrifugation for $30 \mathrm{~s}$ with $12,000 \mathrm{r} / \mathrm{sec}$, the supernatant was used to determine the DNA concentration and purity by 
using the NanoDrop 2000c spectrophotometer (Thermo Scientific, Waltham, MA, USA).

\section{Molecular detection of resistance genes}

Amplification of $v a n A, v a n B, v a n C 1, \operatorname{van} C 2 / 3, v a n M$, and vanD was performed as previously described [11, 12]. Each batch of reactions contained a negative control, a positive control and a blank control. The amplicons were purified using a Qiagen DNA purification kit (Qiagen, Hilden, Germany) and subjected to sequencing. The sequence similarity was determined using the BLAST program from the National Center for Biotechnology Information (http://www.ncbi.nlm.nih.gov/BLAST).

\section{Multi-locus sequence typing (MLST)}

MLST of E. faecium isolates was performed according to the method as previously described [18]. Briefly, the 7 housekeeping genes of $E$. faecium were amplified and sequenced, then strains were assigned to specific STs according to the protocol in MLST database (https:// pubmlst.org/efaecium/).

\section{Statistical analysis}

SPSS statistical software (version 20.0) was used to analyze the data. Difference on the resistance rates between the E. faecalis and E. faecium were analyzed by chi-square test. Chi-square values were corrected when the quantities of VRE isolates were less than 40. Statistical significance was defined when a $P$-value $<0.05$.

\section{Results}

\section{Distribution of clinical enterococci isolates from 2013 to} 2018

Among the 3913 enterococci isolates, E. faecalis $(n=$ $1870,47.8 \%)$ and E. faecium $(n=1738,44.4 \%)$ were the major ones, followed by E. avium $(n=169,4.3 \%)$, E. gallinarum $(n=70,1.8 \%)$, E. casseliflavus $(n=35,0.9 \%), E$. durans $(n=15,0.4 \%)$, E. cecorum $(n=9,0.2 \%)$ and $E$. raffinosus $(n=7,0.2 \%)$. These Enterococcus were mainly isolated from urine specimens $(n=1673,42.8 \%)$, followed by secretions $(n=583,14.9 \%)$, ascites $(n=554$,
$14.2 \%)$, bile $(n=412,10.5 \%)$, blood $(n=273,6.9 \%)$, catheters $(n=169,4.3 \%)$ and others $(n=249,6.4 \%)$.

The annual distribution of Enterococcus isolates is shown in Table 1. It's worthy to note, the proportion of ascite specimens increased annually, from $7.8 \%$ in 2013 to $21.8 \%$ in 2018 , as shown in Table 2.

\section{The susceptibilities of E. faecalis and E. faecium}

The resistance rates of $E$. faecalis and $E$. faecium were shown in Table 3. Overall, E. faecium showed obviously higher resistance rates than those of $E$. faecalis $(P<$ $0.05)$. For $E$. faecium, more than $40 \%$ of the isolates showed resistance toward penicillin G, ampicillin, high concentrations of gentamicin and levofloxacin. Whereas, more than $95 \%$ of them displayed susceptibilities to linezolid, vancomycin, teicoplanin and tigecycline. In detail, from 2013 to 2018, high resistance rates to penicillin G, ampicillin and levofloxacin were shown to be above $80 \%$ every year; Resistance rates to high concentrations of gentamicin decreased yearly, from $60.8 \%$ in 2013 to $27.1 \%$ in 2017 , but rebounded to $38.7 \%$ in 2018; Resistance rates to vancomycin decreased year by year, from $9.3 \%$ in 2013 to $1.4 \%$ in 2018 (Fig. 1a). Whereas, for E. faecalis, an increasing trend of resistance to linezolid was observed, from $0.4 \%$ in 2013 to $4.8 \%$ in 2018; In contrast, resistance rates to penicillin G and ampicillin decreased gradually; Resistance rates to high concentrations of gentamicin and levofloxacin decreased firstly and increased later (Fig. 1b).

\section{Distribution and susceptibilities of VRE}

Screening of VRE showed that 60 VRE, including $58 E$. faecium, 1 E. faecalis and $1 \mathrm{E}$. avium, were included in this study. These VRE were recovered from urine $(n=$ $31)$, catheter $(n=9)$, blood $(n=7)$, ascites $(n=6)$, wound secretions $(n=3)$, bile $(n=2)$, drainage fluid of perirenal abscess $(n=1)$ and hydrothorax $(n=1)$. And the distribution of VRE among every year during 2013-2018 presented a declining trend year by year (Fig. 2a, b).

Sixty VRE isolates showed high resistance to vancomycin (MIC $>256 \mu \mathrm{g} / \mathrm{ml}$ ), and changeable resistance to teicoplanin $(1 \mu \mathrm{g} / \mathrm{ml} \sim>256 \mu \mathrm{g} / \mathrm{ml})$. Fortunately, VRE

Table 1 Annual distribution of Enterococci isolates during 2013-2018

\begin{tabular}{|c|c|c|c|c|c|c|c|c|c|}
\hline Year & E. faecalis & E. faecium & E. avium & E.gallinarum & E.casseliflavus & E. durans & E. cecorum & E.raffinosus & Total \\
\hline 2013 & 263 & 266 & 27 & 2 & 0 & 1 & 1 & 0 & 560 \\
\hline 2014 & 316 & 286 & 17 & 7 & 3 & 2 & 1 & 0 & 632 \\
\hline 2015 & 362 & 265 & 29 & 13 & 9 & 2 & 1 & 2 & 683 \\
\hline 2016 & 305 & 276 & 32 & 17 & 3 & 4 & 3 & 4 & 644 \\
\hline 2017 & 314 & 295 & 30 & 22 & 9 & 2 & 2 & 1 & 675 \\
\hline 2018 & 310 & 350 & 34 & 9 & 11 & 4 & 1 & 0 & 719 \\
\hline Total & 1870 & 1738 & 169 & 70 & 35 & 15 & 9 & 7 & 3913 \\
\hline
\end{tabular}


Table 2 Samples distribution of Enterococci isolates from 2013 to 2018

\begin{tabular}{|c|c|c|c|c|c|c|c|c|c|c|c|c|c|c|}
\hline \multirow[t]{2}{*}{ Samples } & \multicolumn{2}{|l|}{2013} & \multicolumn{2}{|l|}{2014} & \multicolumn{2}{|l|}{2015} & \multicolumn{2}{|l|}{2016} & \multicolumn{2}{|l|}{2017} & \multicolumn{2}{|l|}{2018} & \multicolumn{2}{|l|}{ Total } \\
\hline & Num. & Ratio (\%) & Num. & Ratio (\%) & Num. & Ratio (\%) & Num. & Ratio (\%) & Num. & Ratio (\%) & Num. & Ratio (\%) & Num. & Ratio (\% \\
\hline urine & 256 & 45.7 & 284 & 44.9 & 308 & 45.1 & 266 & 41.3 & 267 & 39.6 & 292 & 40.6 & 1673 & 42.8 \\
\hline ascites & 44 & 7.8 & 56 & 8.9 & 82 & 12 & 92 & 14.3 & 123 & 18.2 & 157 & 21.8 & 554 & 14.2 \\
\hline secretions & 100 & 17.8 & 115 & 18.2 & 106 & 15.5 & 84 & 13 & 98 & 14.5 & 80 & 11.1 & 583 & 14.9 \\
\hline Bile & 62 & 11.1 & 63 & 10 & 66 & 9.7 & 75 & 11.6 & 76 & 11.3 & 70 & 9.7 & 412 & 10.5 \\
\hline blood & 34 & 6.1 & 38 & 6 & 41 & 6 & 51 & 7.9 & 45 & 6.7 & 64 & 8.9 & 273 & 6.9 \\
\hline catheter & 25 & 4.5 & 31 & 4.9 & 30 & 4.4 & 25 & 3.9 & 28 & 4.1 & 30 & 4.2 & 169 & 4.3 \\
\hline others & 39 & 7 & 45 & 7.1 & 50 & 7.3 & 51 & 8 & 38 & 5.6 & 26 & 3.7 & 249 & 6.4 \\
\hline Total & 560 & & 632 & & 683 & & 644 & & 675 & & 719 & & 3913 & 100 \\
\hline
\end{tabular}

strains showed $100 \%$ of susceptibility to linezolid (MIC $\leq 2 \mu \mathrm{g} / \mathrm{ml}$ ) and to fosfomycin.

\section{The prevalence of van genes}

All the 60 VRE strains carried at least one van gene. Among them, 49 strains carried vanA gene (47 vanA in E. faecium, 1 one in E. faecalis and 1 one in E. avium), 10 E. faecium carried vanM gene, and 1 E. faecium carried both $v a n A$ and $v a n M$.

\section{The sequence types of VRE}

Among the 58 VREM isolates, 16 distinct STs were identified, with ST78 $(n=16)$ ST192 $(n=8)$ and ST570 $(n=7)$ being the predominant ones, followed by ST203 $(n=6)$ and ST343 $(n=5) . \operatorname{ST} 414(n=3), \operatorname{ST} 1015(n=2)$, $\operatorname{ST} 17(n=2), \operatorname{ST} 789(n=2), \operatorname{ST} 1014(n=1), \operatorname{ST} 1039(n=$ $1)$ ST18 $(n=1), \operatorname{ST} 202(n=1), \operatorname{ST} 323(n=1)$, ST564 $(n=1)$ and ST80 $(n=1)$ were also found. The distribution of the dominant clones of VRE in 2013-2018 were shown in Table 4. where ST 570 predominated in 2013 and 2015, whereas, ST203 and ST343 were mainly prevalent clones in 2013. Therefore, the epidemic strains spread to our hospital in 2013, and the prevalence of this major clone was scattered and presented in a variety of clonotypes.

\section{Discussion}

The spread of VRE has been a global problem to public health. In this study, we provided data on the characterization including species and specimen distribution of clinical enterococci isolates, as well as prevalence of van genes among VRE in a tertiary hospital based on the data from 2013 to 2018.

We found that the resistance rates of E. faecium toward most of the antimicrobial agents in clinical treatment were higher than those of E. faecalis, especially to penicillin, ampicillin, and levofloxacin. This is in accordance with the previous report [8], providing evidence that $E$. faecium are prone to be more resistant than $E$. faecalis. Albeit that infections caused by E. faecium present a serious clinical challenge for physicians, and treatment options for these infections are limited [8]. Fortunately, good sensitivities toward vancomycin, linezolid, tigecycline and fosfomycin were displayed, which means that there are still potent effective drugs for the treatment of positive cocci, including enterococci.

The high distribution of VREM in our study is consistent with the previous report $[19,20]$, indicating that VREM is the major VRE. Moreover, in our study, VRE strains mainly originated from urine, which was different from the Canadian strains, where VREs primarily

Table 3 Resistance rates (\%) of E. faecalis and E. faecium to antimicrobiol agents

\begin{tabular}{|c|c|c|c|c|}
\hline \multirow[t]{2}{*}{ Antibiotic } & \multirow{2}{*}{$\begin{array}{l}\text { E. faecalis } \\
(n=1870) \\
\text { Resistance rates }(\%)\end{array}$} & $\begin{array}{l}\text { E. faecium } \\
(n=1738)\end{array}$ & \multirow[t]{2}{*}{$x^{2}$} & \multirow[t]{2}{*}{$P$ value } \\
\hline & & Resistance rates (\%) & & \\
\hline penicillin G & 6.8 & 85.6 & 2146.5 & $<0.05$ \\
\hline ampicillin & 2.1 & 83.8 & 2303.3 & $<0.05$ \\
\hline high concentrations of gentamicin & 31 & 41.5 & 42.2 & $<0.05$ \\
\hline levofloxacin & 28.2 & 84 & 1033.1 & $<0.05$ \\
\hline linezolid & 1.9 & 0.4 & 16.3 & $<0.05$ \\
\hline vancomycin & 0.1 & 3.7 & 65.5 & $<0.05$ \\
\hline teicoplanin & 0 & 2.5 & 46.0 & $<0.05$ \\
\hline Tigecycline & 0 & 0 & & $\ldots$ \\
\hline
\end{tabular}




\section{A. The trend of antimicrobial resistance of clinical Enterococcus faecium isolates} to antimicrobial agents during 2013-2018

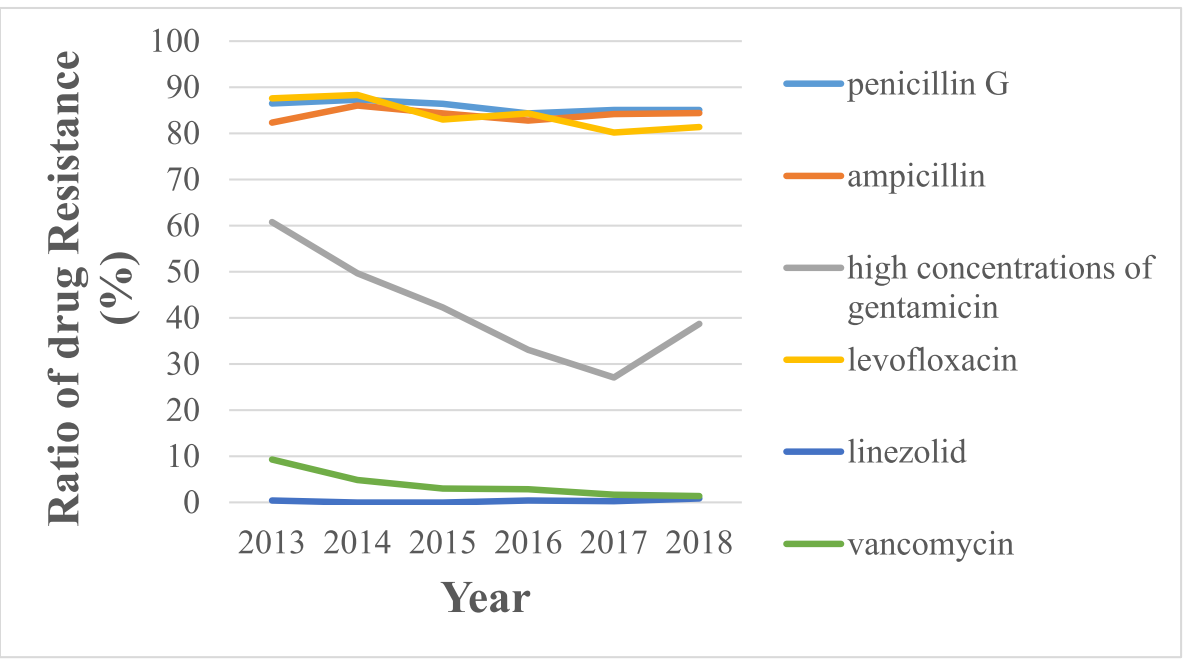

\section{B. The trend of antimicrobial resistance of clinical Enterococcus faecalis isolates}

\section{to antimicrobial agents during 2013-2018}

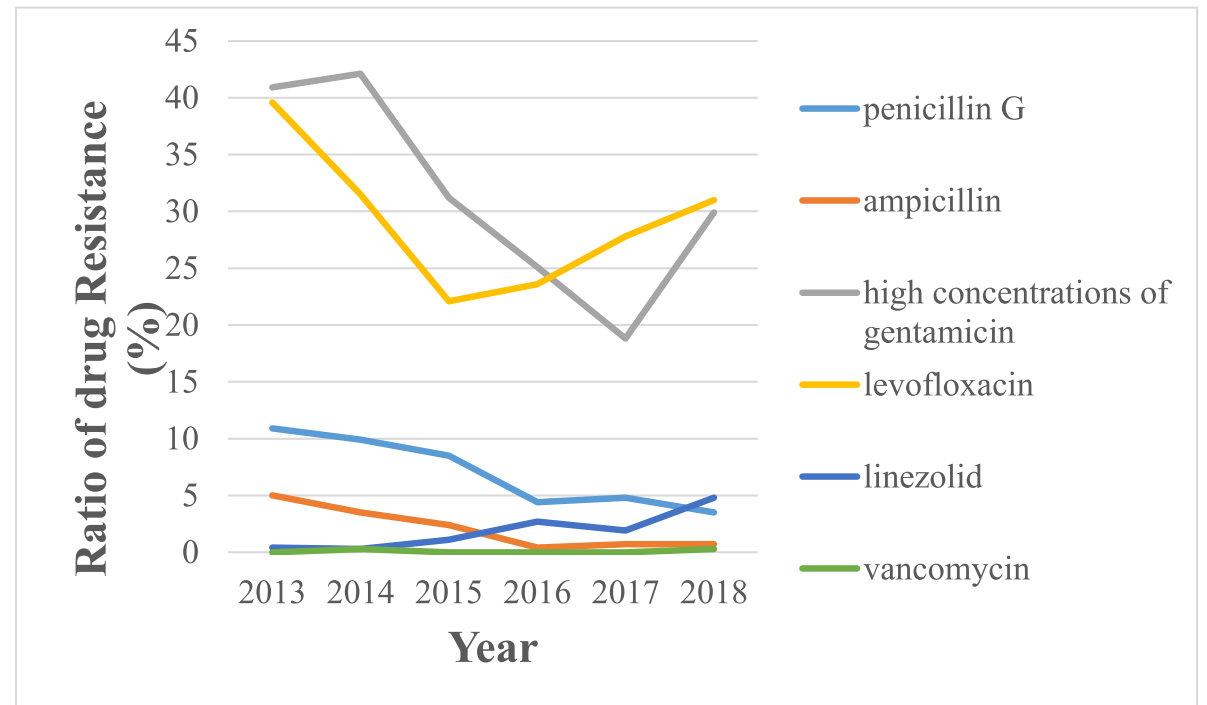

Fig. 1 The trend of antimicrobial resistance of clinical enterococci isolates to antimicrobial agents during 2013-2018: a The trend of antimicrobial resistance of clinical Enterococcus faecium isolates to antimicrobial agents during 2013-2018; b The trend of antimicrobial resistance of clinical Enterococcus faecalis isolates to antimicrobial agents during 2013-2018

originated from blood (68.8\%) [6]. Consistent with previous study (http://www.chinets.com/Data/GermYear), we observed a deceasing trend on the prevalence of VRE year by year, this may depend on the low distribution of VRE in Chinese hospitals [8]. Although it has been increasing steadily since the first description of these organisms in 2010 [21]. So far, the incidence of VRE infections has significantly increased, particularly in parts of Asia, Europe, and the United States [6, 22]. The situation in our study is totally different from the report in U.S. hospitals, where the number of U.S. hospitalizations with VRE discharges more than doubled between 2000 and 2006, with a prevalence as high as 65\% [23]. Noteworthily, a weak upward trend (from $0.4 \%$ in 2013 to $2.4 \%$ in 2018) for linezolid-resistant enterococci was also observed in our study, together with the higher resistance rates of $E$. faecalis to linezolid than those of $E$. faecium, indicating that $E$. faecalis may be easy to develop resistance to linezolid than $E$. faecium, which alert us to carefully use linezolid in the clinical therapy [24]. 


\section{A. The trend on annual number of the vancomycin-resistant enterococci during 2013-2018}

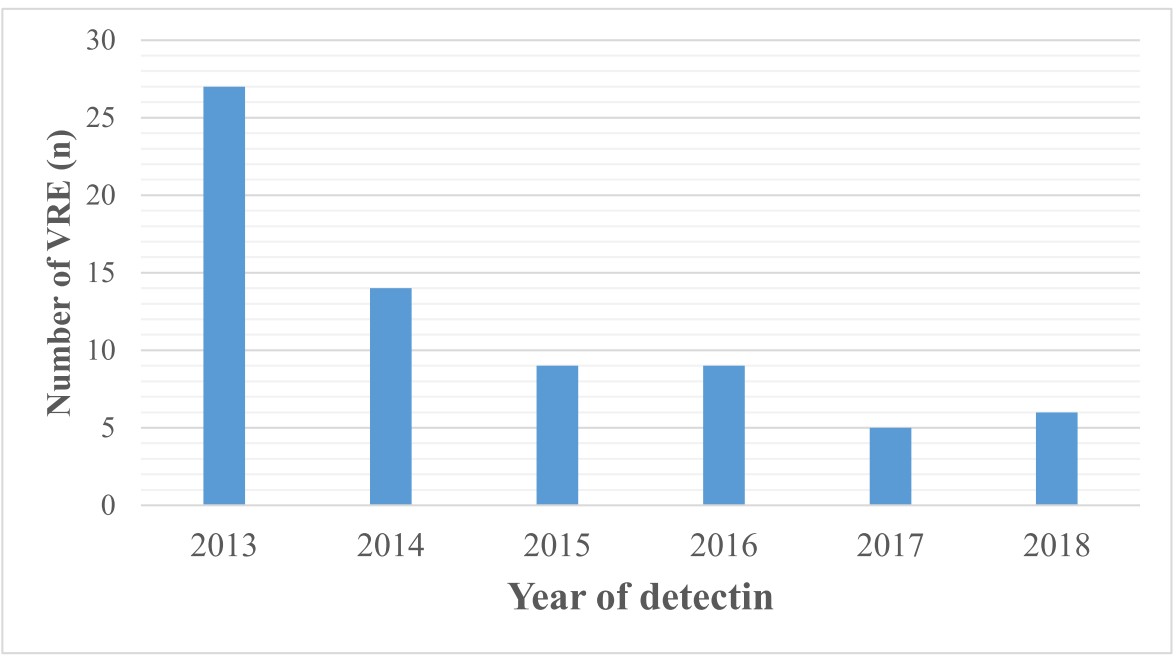

\section{B. The trend on annual proportion of vancomycin-resistant Enterococci in clinical enterococci isolates during 2013-2018}

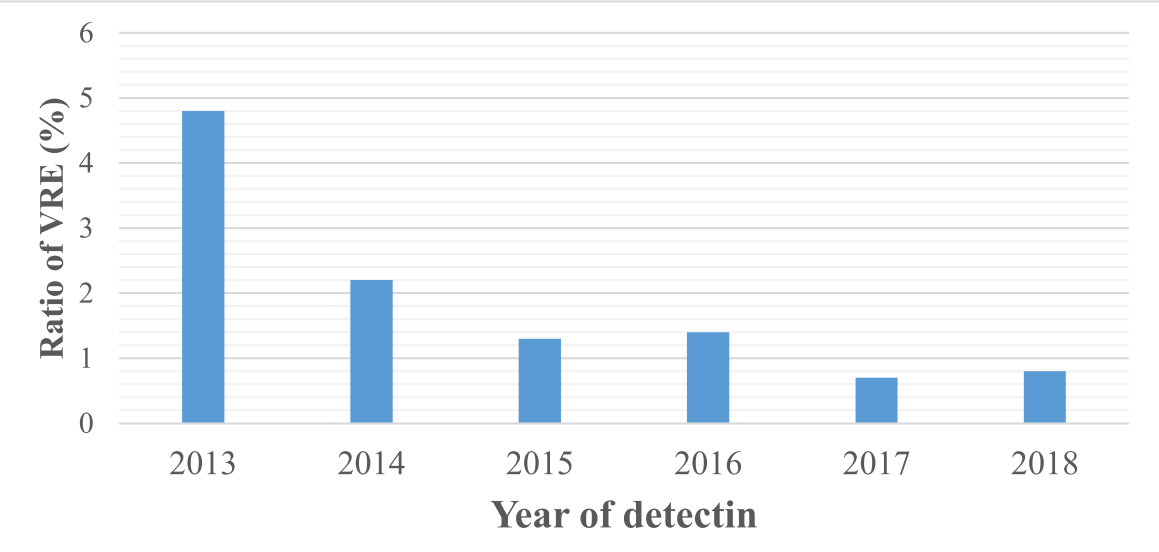

Fig. 2 The trend on annual number/proportion of vancomycin-resistant Enterococci in clinical enterococci isolates during 2013-2018: a The trend on annual number of the vancomycin-resistant enterococci during 2013-2018; $\mathbf{b}$ The trend on annual proportion of vancomycin-resistant enterococci in clinical enterococci isolates during 2013-2018

Even though the high susceptibilities of VRE to linezolid in our study suggest that linezolid is a potentially effective for infections caused by VRE [3].

The high prevalence of vanA gene among VRE has been reported in several Asian countries, mainly South Korea, Japan, and China [25-27], which was also observed in our study. As we know that vanA type has been characterized by acquired resistance to high levels of both vancomycin and teicoplanin $[9,12]$, then we could provide explanation for the high resistance to vancomycin. Furthermore, a similar IS element, IS1216V, has been reported to be widespread among vanA-type VRE, and this element may play an important role in the dissemination of resistance determinants by transposon-mediated fusion of vanA plasmid with other plasmids [28, 29]. Thus, further study may be needed to investigate the distribution of such IS element to explore the probability of dissemination of vanA gene. As we know that, vanM was first identified in Shanghai in 2006 and later found to be the dominant gene mediating resistance to vancomycin in E. faecium [11]. Previous studies showed that vanM gene encodes a 343amino-acid protein that shares $79.9 \%$ amino acid identity with vanA [11]; Phenotypically, vanM-type isolates showed similar antimicrobial susceptibility patterns to the vanA type, with resistance to both vancomycin and teicoplanin [11]. To date, vanM has been identified in 
Table 4 Distribution of van genes and sequence types among vancomycin-resistant Enterococcus faecium

\begin{tabular}{|c|c|c|c|c|c|c|c|c|c|c|}
\hline \multirow{2}{*}{$\begin{array}{l}\text { Sequence } \\
\text { types }\end{array}$} & \multicolumn{6}{|l|}{ Year } & \multicolumn{3}{|c|}{ van genes } & \multirow[t]{2}{*}{ Total } \\
\hline & 2013 & 2014 & 2015 & 2016 & 2017 & 2018 & $\operatorname{van} A$ & vanM & vanA/vanM & \\
\hline 17 & 0 & 2 & 0 & 0 & 0 & 0 & 2 & 0 & 0 & 2 \\
\hline 18 & 1 & 0 & 0 & 0 & 0 & 0 & 1 & 0 & 0 & 1 \\
\hline 78 & 6 & 4 & 2 & 1 & 1 & 2 & 15 & 1 & 0 & 16 \\
\hline 80 & 0 & 1 & 0 & 0 & 0 & 0 & 1 & 0 & 0 & 1 \\
\hline 192 & 1 & 4 & 1 & 0 & 2 & 0 & 3 & 4 & 1 & 8 \\
\hline 202 & 0 & 0 & 1 & 0 & 0 & 0 & 1 & 0 & 0 & 1 \\
\hline 203 & 5 & 1 & 0 & 0 & 0 & 0 & 5 & 1 & 0 & 6 \\
\hline 323 & 0 & 0 & 0 & 0 & 0 & 1 & 1 & 0 & 0 & 1 \\
\hline 343 & 5 & 0 & 0 & 0 & 0 & 0 & 5 & 0 & 0 & 5 \\
\hline 414 & 2 & 1 & 0 & 0 & 0 & 0 & 2 & 1 & 0 & 3 \\
\hline 564 & 0 & 0 & 0 & 1 & 0 & 0 & 0 & 1 & 0 & 1 \\
\hline 570 & 3 & 0 & 3 & 1 & 0 & 0 & 7 & 0 & 0 & 7 \\
\hline 789 & 0 & 0 & 0 & 1 & 1 & 0 & 1 & 1 & 0 & 2 \\
\hline 1014 & 0 & 0 & 0 & 0 & 0 & 1 & 1 & 0 & 0 & 1 \\
\hline 1015 & 1 & 0 & 0 & 0 & 1 & 0 & 2 & 0 & 0 & 2 \\
\hline 1039 & 0 & 0 & 0 & 1 & 0 & 0 & 0 & 1 & 0 & 1 \\
\hline Total & 24 & 13 & 7 & 5 & 5 & 4 & 47 & 10 & 1 & 58 \\
\hline
\end{tabular}

Hangzhou [14, 15], Shanghai [11, 13] and Beijing [16] in China, but was not in other cities of China. In our study, the prevalence of $v a n M$ gene among VRE isolates suggests that this is the first time that we provided the related data in Nanjing, China. Furthermore, we found that all the vanM-containing isolates displayed resistance toward vancomycin, albeit the insertion of an IS1216-like element into the $v a n M$ gene cluster at various positions could lead to the silencing of the VanM phenotype by deletions or partial deletions of $v a n R$, vanS, or $\operatorname{vanX}$ [15]. Additionally, vanM-containing vancomycin-susceptible $E$. faecium strains might switch to a vancomycin-resistant phenotype during prolonged vancomycin treatment, leading to a failure of vancomycin treatment [15]. Therefore, it may be necessary to strengthen the surveillance for $v a n M$ among clinical Enterococcus isolates [30]. More importantly, we also found a co-occurrence of $v a n A$ and $v a n M$ among a VREM, which has been reported previously [16]. To date, the co-existence of $v a n A$ and $v a n C 1$ gene clusters in $E$. gallinarum isolates has been demonstrated to confer highlevel glycopeptide resistance in southern India [31]; An outbreak due to $E$. faecium strain co-carrying $\operatorname{van} A$ and $v a n B$ was reported in France [32]; In addition, vanA-VanD VRE strains were identified in Korea and France [33, 34]. Altogether, the co-occurrence of van genes among single strain indicated the importance of infection control measures.

MLST typing displayed 3 dominant STs, including ST78, ST192 and ST570, which were frequently identified in VREM isolates in China $[13,15,35,36]$. This is quite different from the dominant clone of ST117 and ST80 in Germany [5]. As we know, ST78 has been reported to be the predominant ST among vanA- and vanM-type VREM strains in China [13, 16], although the dominant clone ST78-vanA as the most popular type in our study was mainly separated between 2013 and 2014 (Table 5); ST192 was the most prevalent clone for vanM-type VRE and E. faecium ST192 has been recognized as one of 3 highly prevalent STs responsible for hospital-associated (HA) bloodstream infections in

Table 5 Annual distribution of the combination of sequence types and van genotypes among vancomycin-resistant

\begin{tabular}{llllllll}
\multicolumn{1}{l}{ Enterococcus faecium } \\
\hline Types & 2013 & 2014 & 2015 & 2016 & 2017 & 2018 & Total \\
& $(n=20)$ & $(n=9)$ & $(n=6)$ & $(n=2)$ & $(n=3)$ & $(n=2)$ & \\
\hline ST78-vanA & 5 & 4 & 2 & 1 & 1 & 2 & 15 \\
ST78-vanM & 1 & 0 & 0 & 0 & 0 & 0 & 1 \\
ST192-vanA & 1 & 1 & 0 & 0 & 1 & 0 & 3 \\
ST192-vanM & 0 & 3 & 0 & 0 & 1 & 0 & 4 \\
ST192-vanA/ & 0 & 0 & 1 & 0 & 0 & 0 & 1 \\
vanM & & & & & & & \\
ST570-vanA & 3 & 0 & 3 & 1 & 0 & 0 & 7 \\
ST570-vanM & 0 & 0 & 0 & 0 & 0 & 0 & 0 \\
ST203-vanA & 5 & 0 & 0 & 0 & 0 & 0 & 5 \\
ST203-vanM & 0 & 1 & 0 & 0 & 0 & 0 & 1 \\
ST343-vanA & 5 & 0 & 0 & 0 & 0 & 0 & 5 \\
ST343-vanM & 0 & 0 & 0 & 0 & 0 & 0 & 0 \\
\hline
\end{tabular}


German hospital patients [37]. Strain 4868 with ST192vanA/vanM type detected in our study has also been reported in Beijing, China [16], which alerts us the probability of clone dissemination; In our study, ST570-vanA was mainly isolated in 2013 and 2015; ST343-vanA and ST203-vanA were detected only in 2013, and ST192vanM was distributed in 2014 and 2017 (Table 5), these differences on the distribution of van genes in different clones annually may reflect the prevalent trend of major clone spread, which may relate with the differences in antibiotic use.

Our study has several limitations. Firstly, this is a retrospective study, not all the VRE isolates could be collected for the further analysis; Secondly, this is a single centre study, our data may not reflect all the characterization of VRE isolates from other institutions in China, since the burden of VRE has been shown to vary regionally.

\section{Conclusions}

In conclusion, E. faecalis and E. faecium were the major enterococci strains which are the main pathogens of urinary traction infections. vanA and vanM were the main genes conferring resistance to vancomycin, ST78, ST192 and ST570 were the leading STs of VREM which displayed a decreasing trend year by year. Thus, our findings indicated the importance of performing regional antibiotic resistance surveillance for infection control practices.

\section{Abbreviations \\ VRE: Vancomycin-resistant enterococci; VREM: Vancomycin-resistant Enterococcus faecium; VREF: Vancomycin-resistant Enterococcus faecalis; VREA: Vancomycin-resistant E. avium; MICs: Minimum inhibition concentrations; MLST: Multi-locus sequence typing; STs: Sequence types}

\section{Acknowledgments}

The authors thank the staff of the laboratory of clinical microbiology of Nanjing Drum Tower Hospital, Nanjing, China, for the identification of clinical microorganism.

\section{Authors' contributions \\ WZ, ZH, SH and ZC performed design, contributed to the acquisition, analysis and interpretation of data and drafted the manuscript. SY assisted in the experiment, analysis and coordination of the project. GS, ZY, CX and ZZ participated in strain identification and the MLST analysis. All authors have read and approved the final manuscript.}

\section{Funding}

This study was supported by the Nanjing Medical Science and Technique Development Foundation (Grant No. QRX17143, YKK17056). The funders had no role in study design, data collection and analysis, decision to publish, or preparation of the manuscript.

\section{Availability of data and materials}

The datasets used and/or analysed during the current study are available from the corresponding author on reasonable request.

\section{Ethics approval and consent to participate}

Not applicable.

\section{Consent for publication}

Not applicable.

\section{Competing interests}

The authors declare no conflicts of interest.

Received: 1 February 2020 Accepted: 10 May 2020

Published online: 19 May 2020

\section{References}

1. Reyes K, Bardossy AC, Zervos M. Vancomycin-resistant enterococci: epidemiology, infection prevention, and control. Infect Dis Clin N Am. 2016; 30(4):953-65. https://doi.org/10.1016/j.idc.2016.07.009.

2. MacDougall C, Johnstone J, Prematunge C, Adomako K, Nadolny E, Truong E, Saedi A, Garber G, Sander B. Economic evaluation of vancomycin-resistant enterococci (VRE) control practices: a systematic review [published online ahead of print, 2019 Dec 16]. J Hosp Infect. 2019;S0195-6701(19):30528-6. https://doi.org/10.1016/j.jhin.2019.12.007.

3. Zhou W, Gao S, Xu H, Zhang Z, Chen F, Shen H, Zhang C. Distribution of the optrA gene in Enterococcus isolates at a tertiary care hospital in China. J Glob Antimicrob Resist. 2019;17:180-6. https://doi.org/10.1016/j.jgar.2019. 01.001.

4. ECDC. Surveillance of antimicrobial resistance in Europe. 2016. Available from: https://ecdc.europa.eu/sites/portal/files/documents/AMR-surveillanceEurope-2016.pdf. 2017

5. Markwart R, Willrich N, Haller S, Noll I, Koppe U, Werner G, Eckmanns T, Reuss A. The rise in vancomycin-resistant Enterococcus faecium in Germany: data from the German Antimicrobial Resistance Surveillance (ARS). Antimicrob Resist Infect Control. 2019:8:147. https://doi.org/10.1186/s13756019-0594-3.

6. Simner PJ, Adam H, Baxter M, McCracken M, Golding G, Karlowsky JA, Nichol K, Lagacé-Wiens P, Gilmour MW. Canadian antimicrobial resistance Alliance (CARA), Hoban DJ, Zhanel GG. Epidemiology of vancomycinresistant enterococci in Canadian hospitals (CANWARD study, 2007 to 2013). Antimicrob Agents Chemother. 2015;59(7):4315-7. https://doi.org/10.1128/ AAC.00384-15

7. Li F, Wang Y, Sun L, Wang X. Vancomycin-resistant Enterococcus faecium pneumonia in a uremic patient on hemodialysis: a case report and review of the literature. BMC Infect Dis. 2020;20(1):167. https://doi.org/10.1186/ s12879-020-4892-4.

8. Huang L, Zhang R, Hu Y, Zhou H, Cao J, Lv H, Chen S, Ding S, Chen G. Epidemiology and risk factors of methicillin-resistant Staphylococcus aureus and vancomycin-resistant enterococci infections in Zhejiang China from 2015 to 2017. Antimicrob Resist Infect Control. 2019;8:90. https://doi.org/10. 1186/s13756-019-0539-X.

9. Ahmed MO, Baptiste KE. Vancomycin-resistant enterococci: a review of antimicrobial resistance mechanisms and perspectives of human and animal health. Microb Drug Resist. 2018;24(5):590-606. https://doi.org/10. 1089/mdr.2017.0147.

10. Cattoir $V$, Leclercq R. Twenty-five years of shared life with vancomycinresistant enterococci: is it time to divorce? J Antimicrob Chemother. 2013; 68(4):731-42. https://doi.org/10.1093/jac/dks469.

11. Xu X, Lin D, Yan G, Ye X, Wu S, Guo Y, Zhu D, Hu F, Zhang Y, Wang F, Jacoby GA, Wang $M$. vanM, a new glycopeptide resistance gene cluster found in Enterococcus faecium. Antimicrob Agents Chemother. 2010;54(11): 4643-7. https://doi.org/10.1128/AAC.01710-09.

12. Teo JW, Krishnan $P$, Jureen $R$, Lin RT. Detection of an unusual van genotype in a vancomycin-resistant Enterococcus faecium hospital isolate. J Clin Microbiol. 2011;49(12):4297-8. https://doi.org/10.1128/JCM.05524-11.

13. Chen C, Sun J, Guo Y, Lin D, Guo Q, Hu F, Zhu D, Xu X, Wang M. High prevalence of vanM in vancomycin-resistant Enterococcus faecium isolates from Shanghai, China. Antimicrob Agents Chemother. 2015;59(12):7795-8. https://doi.org/10.1128/AAC.01732-15.

14. Zhang G, Lin X, Cai J, Zhang R, Xiu H, Tian B, Cui W. Characteristics of clinical and environmental vanM-carrying vancomycin-resistant enterococci isolates from an infected patient. Int J Antimicrob Agents. 2018;51(6):932-5. https://doi.org/10.1016/j.ijantimicag.2018.01.003.

15. Sun L, Qu T, Wang D, Chen Y, Fu Y, Yang Q, Yu Y. Characterization of vanM carrying clinical Enterococcus isolates and diversity of the suppressed vanM gene cluster. Infect Genet Evol. 2019;68:145-52. https://doi.org/10.1016/j. meegid.2018.12.015.

16. Sun HL, Liu C, Zhang JJ, Zhou YM, Xu YC. Molecular characterization of vancomycin- resistant enterococci isolated from a hospital in Beijing, China. 
J Microbiol Immunol Infect. 2019;52(3):433-42. https://doi.org/10.1016/j.jmii. 2018.12.008.

17. Clinical and Laboratory Standards Institute. Performance standards for antimicrobial susceptibility testing. 28th ed. CLSI supplement M100. Wayne, PA: CLSI; 2018

18. Homan WL, Tribe D, Poznanski S, Li M, Hogg G, Spalburg E, Van Embden JDA, Willems RJL. Multilocus sequence typing scheme for Enterococcus faecium. J Clin Microbiol. 2002;40(6):1963-71. https://doi.org/10.1128/jcm.40. 6.1963-1971.2002.

19. Kuo AJ, Shu JC, Liu TP, Lu JJ, Lee MH, Wu TS, Su LH, Wu TL. Vancomycinresistant Enterococcus faecium at a university hospital in Taiwan, 2002-2015: fluctuation of genetic populations and emergence of a new structure type of the Tn1546-like element. J Microbiol Immunol Infect. 2018;51(6):821-8. https://doi.org/10.1016/j.jmii.2018.08.008.

20. Chen C, Xu X, Qu T, Yu Y, Ying C, Liu Q, Guo Q, Hu F, Zhu D, Li G, Wang M. Prevalence of the Fosfomycin-resistance determinant, fosB3, in Enterococcus Faecium clinical isolates from China. J Med Microbiol. 2014; 63(Pt 11):1484-9. https://doi.org/10.1099/jmm.0.077701-0.

21. Zhao C, Sun H, Wang H, Liu Y, Hu B, Yu Y, Sun Z, Chu Y, Cao B, Liao K, Lei J, Hu Z, Zhang L, Zhang X, Xu Y, Wang Z, Chen M. Antimicrobial resistance trends among 5608 clinical gram-positive isolates in China: results from the gram-positive Cocci resistance surveillance program (2005-2010). Diagn Microbiol Infect Dis. 2012;73(2):174-81. https://doi.org/10.1016/j. diagmicrobio.2012.03.003.

22. Deshpande LM, Fritsche TR, Moet GJ, Biedenbach DJ, Jones RN. Antimicrobial resistance and molecular epidemiology of vancomycinresistant enterococci from North America and Europe: a report from the SENTRY antimicrobial surveillance program. Diagn Microbiol Infect Dis. 2007; 58(2):163-70. https://doi.org/10.1016/j.diagmicrobio.2006.12.022.

23. Ramsey AM, Zilberberg MD. Secular trends of hospitalization with vancomycin-resistant enterococcus infection in the United States, 20002006. Infect Control Hosp Epidemiol. 2009;30(2):184-6. https://doi.org/10. 1086/593956.

24. Bi R, Qin T, Fan W, Ma P, Gu B. The emerging problem of linezolid-resistant Enterococcus. J Glob Antimicrob Resist. 2018;13:11-9. https://doi.org/10. 1016/j.jgar.2017.10.018.

25. Lee WG, Huh JY, Cho SR, Lim YA. Reduction in glycopeptide resistance in vancomycin-resistant enterococci as a result of vanA cluster rearrangements. Antimicrob Agents Chemother. 2004;48(4):1379-81. https:// doi.org/10.1128/aac.48.4.1379-1381.2004.

26. Park IJ, Lee WG, Shin JH, Lee KW, Woo GJ. VanB phenotype-vanA genotype Enterococcus faecium with heterogeneous expression of teicoplanin resistance. J Clin Microbiol. 2008;46(9):3091-3. https://doi.org/10.1128/JCM. 00712-08.

27. Lauderdale TL, McDonald LC, Shiau YR, Chen PC, Wang HY, Lai JF, Ho M. Vancomycin-resistant enterococci from humans and retail chickens in Taiwan with unique VanB phenotype-vanA genotype incongruence. Antimicrob Agents Chemother. 2002;46(2):525-7. https://doi.org/10.1128/ aac.46.2.525-527.2002.

28. Darini AL, Palepou MF, Woodford N. Effects of the movement of insertion sequences on the structure of VanA glycopeptide resis- tance elements in Enterococcus faecium. Antimicrob Agents Chemother. 2000;44(5):1362-4. https://doi.org/10.1128/aac.44.5.1362-1364.2000.

29. Palepou MF, Adebiyi AM, Tremlett CH, Jensen LB, Woodford N. Molecular analysis of diverse elements mediating VanA glycopeptide resistance in enterococci. J Antimicrob Chemother. 1998;42(5):605-12. https://doi.org/10. 1093/jac/42.5.605.

30. Sun L, Chen Y, Hua X, Chen Y, Hong J, Wu X, Jiang Y, Cchaik W, Quu T, Yu $Y$. Tandem amplification of the vanM gene cluster drives vancomycin resistance in vancomycin-variable enterococci [published online ahead of print, 2019 Nov 19]. J Antimicrob Chemother. 2019:dkz461. https://doi.org/ 10.1093/jac/dkz461.

31. Praharaj I, Sujatha S, Parija SC. Phenotypic \& genotypic characterization of vancomycin resistant Enterococcus isolates from clinical specimens. Indian J Med Res. 2013;138(4):549-56 PMCID: PMC3868069.

32. Marcadé G, Micol JB, Jacquier H, Raskine L, Donay J-L, Nicolas-Viaud S, Rouveau M, Ribaud P, Dombret H, Leclercq R, Cambau E. Outbreak in a haematology unit involving an unusual strain of glycopeptide-resistant Enterococcus faecium carrying both vanA and vanB genes. J Antimicrob Chemother. 2014;69(2):500-5. https://doi.org/10.1093/jac/dkt376.
33. Naas T, Fortineau N, Snanoudj R, Spicq C, Durrbach A, Nordmann P. First nosocomial outbreak of vancomycin-resistant Enterococcus faecium expressing a VanD-like phenotype associated with a vanA genotype. J Clin Microbiol. 2005;43(8):3642-9. https://doi.org/10.1128/JCM.43.8.3642-3649. 2005

34. Song JH, Ko KS, Suh JY, Oh WS, Kang C, Chung DR, Peck KR, Lee NY, Lee WG. Clinical implications of vancomycin-resistant Enterococcus faecium (VRE) with VanD phenotype and vanA genotype. J Antimicrob Chemother. 2008;61(4):838-44. https://doi.org/10.1093/jac/dkn025.

35. Zhu X, Zheng B, Wang S, Willems RJL, Xue F, Cao X, Bo S, Liu J. Molecular characterisation of outbreak-related strains of vancomycin-resistant Enterococcus faecium from an intensive care unit in Beijing, China. J Hosp Infect. 2009;72(2):147-54. https://doi.org/10.1016/j.jhin.2009.02.014.

36. Zheng B, Tomita H, Xiao YH, Wang S, Li Y, lke Y. Molecular characterization of vancomycin-resistant enterococcus faecium isolates from mainland China. J Clin Microbiol. 2007;45(9):2813-8. https://doi.org/10.1128/JCM. 00457-07.

37. Werner G, Klare I, Fleige C, Gernger U, Witte W, Just HM, Ziegler R. Vancomycin-resistant vanB-type Enterococcus faecium isolates expressing varying levels of vancomycin resistance and being highly prevalent among neonatal patients in a single ICU. Antimicrob Resist Infect Control. 2012;1(1): 21. https://doi.org/10.1186/2047-2994- 1-21.

\section{Publisher's Note}

Springer Nature remains neutral with regard to jurisdictional claims in published maps and institutional affiliations.
Ready to submit your research? Choose BMC and benefit from:

- fast, convenient online submission

- thorough peer review by experienced researchers in your field

- rapid publication on acceptance

- support for research data, including large and complex data types

- gold Open Access which fosters wider collaboration and increased citations

- maximum visibility for your research: over $100 \mathrm{M}$ website views per year

At BMC, research is always in progress.

Learn more biomedcentral.com/submissions 